\title{
吡唑环桥连简约型长春碱类似物的设计合成
}

\author{
国翠平 ${ }^{a} \quad$ 彭群龙 $^{a} \quad$ 潘 龙 ${ }^{a} \quad$ 张冬梅 ${ }^{a, b}$ 陈河如*, $a, b$ \\ ( ${ }^{a}$ 暨南大学药学院中药及天然药物研究所 广州 510632) \\ $(b$ 广东省中药药效物质基础及创新药物研究重点实验室 广州 510632)
}

\begin{abstract}
摘要 利用焦磷酰氯 $\left(\mathrm{P}_{2} \mathrm{O}_{3} \mathrm{Cl}_{4}\right)$ 和 DMF 为 Vilsmeier-Haack 试剂, 与 2,3,3-三甲基-3H-㖿哚反应生成二甲酰化中间体化合 物, 再与肤衍生物反应生成系列吡唑环桥连简约型长春碱类似物 $\mathbf{2 a} \sim \mathbf{2 m}$. 目标化合物的结构均经 ${ }^{1} \mathrm{H} N \mathrm{NMR},{ }^{13} \mathrm{C} N \mathrm{NM}$ 和 HRMS 确证. 初步的抗肿瘤活性数据表明, 在 $50 \mu \mathrm{mol} / \mathrm{L}$ 的浓度下, 大部分目标化合物对人乳腺癌细胞株(MCF-7) (estrogen-positive)和人肝癌细胞株(HepG2)具有一定的抗肿瘤活性, 其中 $\mathbf{2 f}$ 和 $\mathbf{2 j}$ 抗肿瘤作用较强, 它们对 MCF-7 细胞 株的存活率分别为 $28.0 \%$ 和 $21.4 \%$; 而对 HepG2 细胞株的存活率分别为 $31.6 \%$ 和 $34.0 \%$.
\end{abstract}

关键词＼cjkstart药核相似性；简约设计；长春碱类似物；抗肿瘤；药物合成

\section{Design and Synthesis of Pyrazol-Bridged Simplified Vinblastine Analogues}

\author{
Guo, Cuiping ${ }^{a} \quad$ Peng, Qunlong ${ }^{a} \quad$ Pan, Long $^{a} \quad$ Zhang, Dongmei ${ }^{a, b} \quad$ Chen, Heru*,a,b \\ ( ${ }^{a}$ Institute of Traditional Chinese Medicine and Natural Products, College of Pharmacy, \\ Jinan University, Guangzhou 510632) \\ ( ${ }^{b}$ Guangdong Province Key Laboratory of Pharmacodynamic Constituents of Traditional Chinese Medicine and \\ New Drugs Research, Guangzhou 510632)
}

\begin{abstract}
A series of pyrazol-brigded simplified vinblastine analogues $\mathbf{2 a} \sim \mathbf{2} \mathbf{m}$ have been synthesized by the reaction of hydrazine derivatives with diformyl intermediates, which were prepared from 2,3,3-trimethyl-3H-indoles using $\mathrm{P}_{2} \mathrm{O}_{3} \mathrm{Cl}_{4} / \mathrm{DMF}$ as Vilsmeier-Haack reagent. All the target compounds have been identified by ${ }^{1} \mathrm{H}$ NMR, ${ }^{13} \mathrm{C}$ NMR and HRMS. The preliminary results of anticancer tests indicated that most of the compounds exhibit certain extent of anticancer activity against both MCF-7 (estrogen-positive) and HepG2 cell lines at the concentration of $50 \mu \mathrm{mol} / \mathrm{L}$, respectively. $\mathbf{2} \mathbf{f}$ and $\mathbf{2 j}$ are the two best active compounds, where their cell viabilities against MCF-7 cell line are $28.0 \%$ and $21.4 \%$, respectively; while against HepG2 cell line, $31.6 \%$ and $34.0 \%$, respectively.

Keywords pharmacophore similarity; simplified design; vinca alkaloids analogues; antitumor; drug synthesis
\end{abstract}

长春碱类化合物是临床上重要的抗肿瘤药物 ${ }^{[1 \sim 4]}$, 但在长春花中含量很低, 加上其结构复杂, 难以实现工 业化全合成, 这些因素制约了长春碱类药物在临床上的 实际应用. 长期以来, 人们一致致力于寻找抗肿瘤活性 高、系统毒性低的长春碱衍生物, 大部分工作主要集中 在对长春碱类化合物的局部结构改造和修饰 ${ }^{[5 \sim 7]}$, 这些 衍生物仍然具有长春碱类化合物复杂的稠杂环结构特 征. 因此, 在保留长春碱类化合物的抗肿瘤活性及机制 的前提下, 最大程度地简化长春碱类化合物的结构是另 一个有价值的研究方向. Roussi 等 ${ }^{[8]}$ 在这方面做了初步
尝试, 他们将长春碱中的文朵灵环用简单的芳环结构替 换, 合成了部分简化的长春碱类似物, 但仍然保留长春 质碱的基本骨架，目标化合物的分子结构仍然比较复 杂.

本课题组提出 “药核相似性” 原则, 即药核结构相 似的化合物可能具有相似的生物功能. 在前期工作中设 计合成了一系列乙烯基桥连的长春碱类似物，如图 1 所示 ${ }^{[9]}$. 通过实验证明该类化合物仍然部分保留了长春 碱的抗肿瘤活性, 且与长春碱具有相似的抗肿瘤作用机 制. 为了拓展结构多样性，尝试以吡唑环代替乙烯基作

* E-mail: thrchen@jnu.edu.cn

Received July 29, 2014; revised August 27, 2014; published online August 29, 2014.

Project supported by the National Natural Science Foundation of China (No. 81172982).

国家自然科学基金(No. 81172982)资助项目. 
为桥连结构合成系列新的简约型长春碱类似物, 并测定 该类化合物对人乳腺癌细胞株(MCF-7) (estrogenpositive)和人肝癌细胞株(HepG2)的抗肿瘤活性, 通过探 讨其结构-活性关系(structure-activity relationship, SAR), 夯实 “药核相似性” 原则的科学内涵, 为进一步设计合 成抗肿瘤活性更好的简约型长春碱类似物提供实验依 据.

\section{1 结果与讨论}

\section{1 吡唑环桥连简约型长春碱类似物的合成}

参考文献[10, 11]方法, 2,3,3-三甲基-3H-吲哚通过 维尔斯迈尔-哈克反应(Vilsmeier-Haack)在 2-甲基上引 入两个甲酰基, 生成中间产物 1, 1 与肼衍生物反应生成
系列目标化合物 $\mathbf{2 a} \sim \mathbf{2 m}$ (Scheme 1). 传统上, Vilsmeier Haack 试剂由三氯氧磷 $\left(\mathrm{POCl}_{3}\right)$ 与 $N, N$-二甲基甲酰胺 (DMF)反应制备. 但由于 $\mathrm{POCl}_{3}$ 是剧毒化学品，属国家 管控的危险品，在一定程度上限制了该方法的应用. 文 献[12]中有报道, 可以用草酰氯 $\left[(\mathrm{COCl})_{2}\right]$ 和二氯亚砜 $\left(\mathrm{SOCl}_{2}\right)$ 替代 $\mathrm{POCl}_{3}$ 作为无机酸性卤化剂. 通过探索, 本 文发现用焦磷酰氯 $\left(\mathrm{P}_{2} \mathrm{O}_{3} \mathrm{Cl}_{4}\right)$ 替代 $\mathrm{POCl}_{3}$, 与 $\mathrm{DMF}$ 反应生 成 Vilsmeier-Haack 试剂的效果最佳.

利用 $\mathrm{P}_{2} \mathrm{O}_{3} \mathrm{Cl}_{4} / \mathrm{DMF}$ 试剂对 2,3,3-三甲基-3H-吲哚的 2-甲基进行甲酰化，顺利合成中间体化合物 1, 收率 $90 \%$. 该甲酰化反应的可能机理如 Scheme 2 所示. 在冰 浴条件下, $\mathrm{DMF}$ 与 $\mathrm{P}_{2} \mathrm{O}_{3} \mathrm{Cl}_{4}$ 反应生成加合物卤代亚甲基
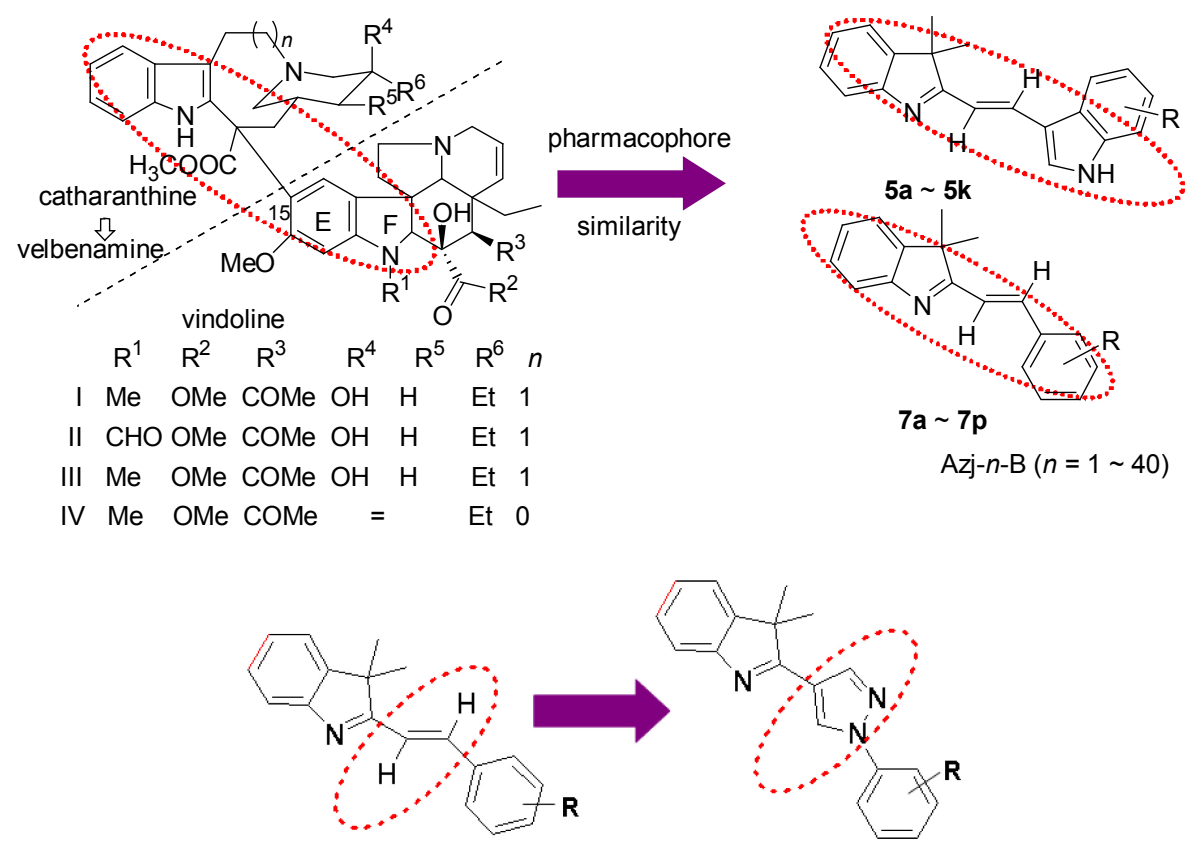

图 1 基于药核相似性的系列长春碱类似物的结构图

Figure 1 Structures of simplified vinca alkaloids based on pharmacophore similarity
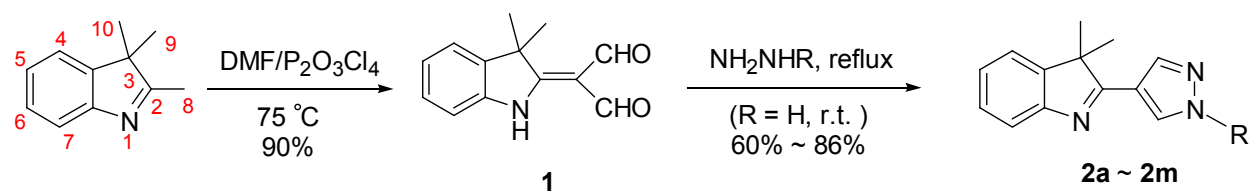

\begin{tabular}{|c|c|c|c|c|c|}
\hline Entry & $\mathrm{R}$ & Yield/\% & Entry & $\mathrm{R}$ & Yield/\% \\
\hline $2 \mathbf{a}$ & $\mathrm{H}$ & 85 & $2 \mathrm{~h}$ & $3-\mathrm{NO}_{2} \mathrm{C}_{6} \mathrm{H}_{4}$ & 62 \\
\hline $2 \mathrm{~b}$ & $\mathrm{C}_{6} \mathrm{H}_{5}$ & 85 & $2 \mathbf{i}$ & $2,4-\left(\mathrm{NO}_{2} \mathrm{C}_{6} \mathrm{H}_{3}\right)_{2}$ & 60 \\
\hline $2 c$ & $3-\mathrm{MeC}_{6} \mathrm{H}_{4}$ & 86 & $2 j$ & $2-\mathrm{CF}_{3} \mathrm{C}_{6} \mathrm{H}_{4}$ & 85 \\
\hline 2d & 4- $\mathrm{MeC}_{6} \mathrm{H}_{4}$ & 86 & $2 k$ & $3-\mathrm{CF}_{3} \mathrm{C}_{6} \mathrm{H}_{4}$ & 86 \\
\hline $2 e$ & $3-\mathrm{MeOC}_{6} \mathrm{H}_{4}$ & 86 & 21 & $4-\mathrm{CF}_{3} \mathrm{C}_{6} \mathrm{H}_{4}$ & 60 \\
\hline $2 f$ & $4-\mathrm{MeOC}_{6} \mathrm{H}_{4}$ & 86 & $2 m$ & $3,5-\left(\mathrm{CF}_{3} \mathrm{C}_{6} \mathrm{H}_{3}\right)_{2}$ & 60 \\
\hline $2 \mathrm{~g}$ & $2 \mathrm{NO}_{2} \mathrm{C}_{6} \mathrm{H}_{4}$ & 67 & & & \\
\hline
\end{tabular}


铵盐, 即 Vilsmeier-Haack 试剂. 该试剂是弱的亲电试 剂，与 2,3,3-三甲基-3H-吲哚发生类 Aldol 反应，随后消 去 $\mathrm{Cl}$ 、水亲核加成后发生电子重排生成 1.1 与肼类衍生 物反应成吡唑环得目标化合物 $\mathbf{2 a} \sim 2 \mathbf{m}$, 收率 $60 \%$ $86 \%$ (Scheme 2).
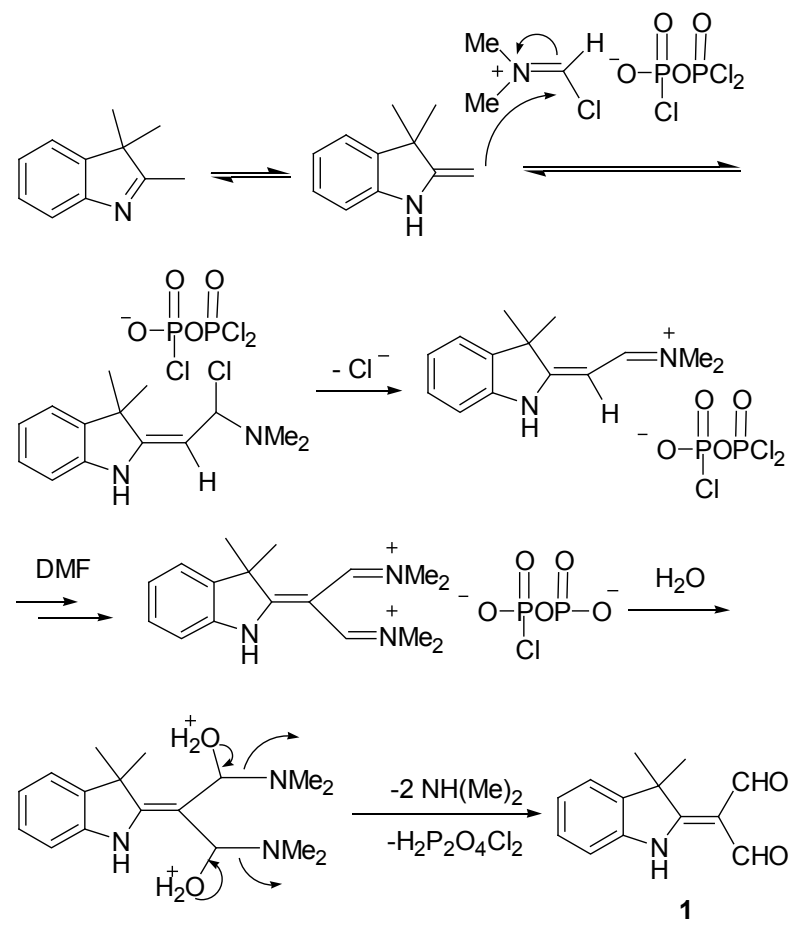

Scheme 2

显而易见，中间体化合物 $\mathbf{1}$ 与胇衍生物的反应与取 代基 $\mathrm{R}$ 的电子属性相关. 当 $\mathrm{R}$ 为富电子体系, 如为 $\mathrm{H}$ 或 其芳环上有给电子诱导效应或共轭效应的基团时 (2a 2f), 反应收率较高, 为 $85 \% \sim 86 \%$; 当 $\mathrm{R}$ 为缺电子体系, 如其芳环上有强的吸电子共轭效应的基团时 $(\mathbf{2 g} \sim \mathbf{2 i})$, 反应收率较低, 为 $60 \% \sim 67 \%$; 当 $\mathrm{R}$ 芳环上的强吸电子 共轭效应基团处于邻/间位时, 对反应的活性影响很小 $(2 \mathbf{j}$ 和 $\mathbf{2 k})$, 其收率与 $\mathbf{2 a} \sim \mathbf{2 f}$ 相当; 当 $\mathrm{R}$ 芳环上的吸电子 诱导基团处于对位时(2i), 则对反应收率的影响较大, 只有 $60 \%$.

\section{2 吡唑环桥连简约型长春碱类似物的抗肿瘤作用初 步研究}

含吡唑环化合物具有广泛的生物活性, 包括抗肿瘤 活性 ${ }^{[13]}$. 参考文献[14]方法, 利用噻唑蓝(MTT)法测定 了本文报道的系列吡唑环桥连简约型长春碱类似物 2a $\sim 2 m$ 对人乳腺癌细胞株 MCF-7 (estrogen-positive)和 人肝癌细胞株 $\mathrm{HepG} 2$ 的抗细胞增殖抑制活性, 以长春 碱作为阳性对照药. 结果如表 1 所示, 当化合物的浓度 为 $5 \mu \mathrm{mol} / \mathrm{L}$ 时, 目标化合物对两种细胞株均没有抗肿瘤 活性, MCF-7 和 HepG2 细胞株的存活率均在 87\% 98\%
之间. 当化合物浓度增加至 $50 \mu \mathrm{mol} / \mathrm{L}$ 时, 大部分化合 物对两种细胞株均显示出不同程度的活性. 其中 $2 \mathbf{f}$ 和 $2 \mathbf{j}$ 具有较强的活性，对 MCF-7 细胞存活率分别为 $28.0 \%$ 和 $31.6 \%$; 而对 HepG2 细胞的存活率分别为 $21.4 \%$ 和 $34.0 \%$. 化合物 $2 \mathrm{e}, \mathbf{2 i}, \mathbf{2 k}$ 和 $2 \mathrm{I}$ 同样表现出一定程度的抗 肿瘤活性，其细胞存活率在 39.1\% 66.3\%之间.

表 1 目标化合物 $\mathbf{2} \mathbf{a} \sim \mathbf{2 m}$ 的抗肿瘤活性数据(存活率 $/ \%)^{a}$

Table 1 Anticancer activity of compounds $\mathbf{2 a} \sim \mathbf{2 m}$ (viability rate/\%)

\begin{tabular}{lccccc}
\hline \multirow{2}{*}{ Entry } & \multicolumn{2}{c}{ MCF-7 } & & \multicolumn{2}{c}{ HepG2 } \\
\cline { 2 - 3 } \cline { 5 - 6 } & $50 \mu \mathrm{mol} / \mathrm{L}$ & $5 \mu \mathrm{mol} / \mathrm{L}$ & & $50 \mu \mathrm{mol} / \mathrm{L}$ & $5 \mu \mathrm{mol} / \mathrm{L}$ \\
\hline $\mathbf{2 a}$ & $90.4 \pm 5.0$ & $96.3 \pm 5.0$ & & $97.6 \pm 2.4$ & $98.3 \pm 3.7$ \\
$\mathbf{2 b}$ & $75.6 \pm 4.7$ & $97.5 \pm 3.7$ & & $65.0 \pm 4.4$ & $94.4 \pm 0.8$ \\
$\mathbf{2 c}$ & $77.4 \pm 3.4$ & $98.3 \pm 1.3$ & & $55.4 \pm 7.9$ & $91.7 \pm 5.6$ \\
$\mathbf{2 d}$ & $74.3 \pm 5.4$ & $98.9 \pm 3.5$ & & $74.9 \pm 3.1$ & $97.9 \pm 5.3$ \\
$\mathbf{2 e}$ & $52.6 \pm 3.3$ & $98.2 \pm 6.2$ & & $61.1 \pm 3.3$ & $87.4 \pm 4.1$ \\
$\mathbf{2 f}$ & $28.0 \pm 1.2$ & $66.9 \pm 9.1$ & & $31.6 \pm 1.5$ & $94.0 \pm 2.9$ \\
$\mathbf{2 g}$ & $82.6 \pm 1.4$ & $97.9 \pm 6.1$ & & $52.9 \pm 7.9$ & $92.5 \pm 7.7$ \\
$\mathbf{2 h}$ & $86.0 \pm 2.6$ & $94.1 \pm 2.2$ & & $83.6 \pm 6.6$ & $93.6 \pm 4.7$ \\
$\mathbf{2 i}$ & $41.4 \pm 1.2$ & $98.1 \pm 2.7$ & & $41.0 \pm 4.0$ & $91.8 \pm 3.4$ \\
$\mathbf{2 j}$ & $21.4 \pm 0.1$ & $89.4 \pm 9.3$ & & $34.0 \pm 0.1$ & $89.3 \pm 3.0$ \\
$\mathbf{2 k}$ & $47.6 \pm 4.7$ & $92.7 \pm 3.9$ & & $66.3 \pm 2.8$ & $88.0 \pm 0.7$ \\
$\mathbf{2 l}$ & $39.1 \pm 5.5$ & $83.0 \pm 1.5$ & & $59.5 \pm 1.2$ & $98.9 \pm 3.5$ \\
$\mathbf{2 m}$ & $67.7 \pm 1.8$ & $93.2 \pm 4.0$ & & $96.1 \pm 9.6$ & $96.6 \pm 0.5$ \\
长春碱 & $18.1 \pm 5.1$ & $28.1 \pm 5.6$ & $22.1 \pm 0.9$ & $23.8 \pm 1.6$ \\
\hline
\end{tabular}

${ }^{a}$ Values are shown as means \pm S.D. from at least three independent experiments.

从表 1 可见, $\mathrm{R}$ 芳环上取代基的电子性质对抗肿瘤 作用没有表现出明显的影响, 但其在芳环上的相对位置 对抗肿瘤活性有影响. 当取代基处于 4-和 2-位时, 抗肿 瘤活性较强. 相对而言, 芳环上 4-或 2-位上大的取代基 有利于增强其抗癌活性.

可见，吡唑环桥连简约型长春碱类似物仍然保留有 一定的抗肿瘤活性 $\left(5 \mu \mathrm{mol} / \mathrm{L}<\mathrm{IC}_{50}<50 \mu \mathrm{mol} / \mathrm{L}\right)$, 但活 性较乙烯桥连简约型长春碱类似物弱 $\left(\mathrm{IC}_{50}=0.89\right.$ $\mu \mathrm{mol} / \mathrm{L}$ ). 究其原因，与该类化合物的抗肿瘤作用机制 有关. Gigant 等 ${ }^{[15]}$ 对长春碱类化合物抗肿瘤的分子作用 机制的研究结果表明，长春碱化合物分子像 “楔子”一 样作用在两个微管蛋白分子之间，抑制微管蛋白的聚 集，使纺锤体不能形成，使细胞分裂停止在有丝分裂中 期. 显然, 长春碱类化合物与微管蛋白的相互作用取决 于该分子在三维空间的构象、分子的大小及与微管蛋白 的相互作用力等. 从其三维结构上看, 乙烯桥连简约型 长春碱类似物更好地模拟长春碱的片层三维空间构象, 因而表现出较强的与微管蛋白 “楔合” 的作用; 而 $3(N)$-位芳基取代的吡唑环桥连简约型长春碱类似物更 像 V 型结构, 不利于与微管蛋白的 “楔合”。该结果为 今后设计活性更好的简约型长春碱类似物提供科学依 据. 


\section{2 结论}

本文成功合成了 13 个吡唑环桥连简约型长春碱类 似物 2a $2 \mathbf{m}$, 收率 60\% 86\%, 并进一步研究了该类化 合物的抗肿瘤活性. 结果表明, 在浓度为 $50 \mu \mathrm{mol} / \mathrm{L}$ 时, 大部分化合物对人乳腺癌细胞株(MCF-7) (estrogenpositive)和人肝癌细胞株(HepG2)两种细胞株均表现出 不同程度的抗增殖抑制活性, 其中 $2 \mathbf{f}$ 和 $2 \mathbf{j}$ 是活性最强 的两个化合物, 它们对 MCF-7 细胞株的存活率分别为 $28.0 \%$ 和 $21.4 \%$; 而对 HepG2 细胞株的存活率分别为 $31.6 \%$ 和 $34.0 \%$.

\section{3 实验部分}

\section{1 仪器与试剂}

Bruker-AV400MHz 型核磁共振仪(瑞士 Bruker 公 司); LC-UV100 液相色谱仪(上海伍丰科学仪器有限公 司); 柱层析硅胶(200 300 目, 烟台化学工业研究所); 薄层层析硅胶(HSGF-254, 烟台江友硅胶开发有限公 司); 人乳腺癌细胞株( MCF-7) (estrogen-positive)和人肝 癌细胞株 (HepG2) 购买于美国典型培养物保藏中心 (ATCC, Rockville, MD); 其它化学品均为市售国产分析 纯试剂, 不经纯化, 直接使用.

\section{2 实验方法}

\subsubsection{2-(2,3,3-三甲基-3H-吲哚基)丙二醛(1)的合成}

$5 \mathrm{~mL} \mathrm{~N}, N$-二甲基甲酰胺(DMF)在冰浴中冷却, 滴 加焦磷酰氯 $5.5 \mathrm{~mL}$ (37.5 mmol), 搅拌 $10 \mathrm{~min}$. 随后滴加 $2.0 \mathrm{~mL}$ (12.5 mmol) 2,3,3-三甲基- $3 H$-吲哚溶解于 $5 \mathrm{~mL}$ 的 DMF 溶液. 滴加完毕, 移去冰浴, 加热到 $75^{\circ} \mathrm{C}$, 反应 $4 \sim 6$ h. 将反应混合物滴加到冰水中, 用 $35 \% \mathrm{NaOH}$ 调 节 $\mathrm{pH}$ 值至中性. 乙酸乙酯萃取 $(100 \mathrm{~mL} \times 3)$, 合并有机 相, 水洗涤数次, 无水 $\mathrm{MgSO}_{4}$ 干燥. 旋干, 乙醇重结晶 得橙黄色固体 $2.4 \mathrm{~g}$, 收率 $90 \% .{ }^{1} \mathrm{H}$ NMR $\left(\mathrm{CDCl}_{3}, 400\right.$ $\mathrm{MHz}) \delta: 1.76(\mathrm{~s}, 6 \mathrm{H}), 7.21(\mathrm{t}, J=8.0 \mathrm{~Hz}, 1 \mathrm{H}), 7.29 \sim 7.32$ (m, 1H), 7.35 (dt, $J=4.0,12.0 \mathrm{~Hz}, 2 \mathrm{H}), 9.78$ (s, 2H), 13.58 $(\mathrm{s}, 1 \mathrm{H}) ;{ }^{13} \mathrm{C}$ NMR $\left(75 \mathrm{MHz}, \mathrm{CDCl}_{3}\right) \delta: 23.4(2), 51.4$, $109.4,112.7,122.2,125.8,128.3,139.3,140.7,179.5(2)$, 190.2; ESI-MS $m / z: 216.3[\mathrm{M}+\mathrm{H}]^{+}, 214.5[\mathrm{M}-\mathrm{H}]^{-}$. 数 据同文献[10].

\subsection{2 吡唑桥连简约型长春碱类似物的合成}

2-(1H-吡唑-基)-3,3-二甲基-3H-吲哚(2a)的合成：将 $43 \mathrm{mg}(0.2 \mathrm{mmol})$ 中间体化合物 $\mathbf{1}$ 溶解于 $5 \mathrm{~mL}$ 无水乙醇 中, 加入 $80 \%$ 水合肼 $25 \mu \mathrm{L}(0.4 \mathrm{mmol})$, 室温搅拌 $24 \mathrm{~h}$. 反应混合物浓缩, 过滤, 乙醇重结晶, 得到针状黄色晶 体 2a $33 \mathrm{mg}$, 收率 $85 \% .{ }^{1} \mathrm{H} \mathrm{NMR}\left(\mathrm{CDCl}_{3}, 400 \mathrm{MHz}\right) \delta$ : 1.52 (s, 6H), 7.24 (t, $J=8.0 \mathrm{~Hz}, 2 \mathrm{H}), 7.35$ (dd, $J=4.0,8.0$
$\mathrm{Hz}, 2 \mathrm{H}), 7.64(\mathrm{~d}, J=8.0 \mathrm{~Hz}, 1 \mathrm{H}), 8.33(\mathrm{~s}, 2 \mathrm{H}) ;{ }^{13} \mathrm{C} \mathrm{NMR}$ $\left(75 \mathrm{MHz}, \mathrm{CDCl}_{3}\right) \delta:$ 26.2(2), 54.7, 117.5, 121.3, 122.4, 126.8(2), 129.3, 135.4, 147.5, 154.7, 180.4. ESI-MS 212.2 $[\mathrm{M}+\mathrm{H}]^{+}$; HRMS $m / z$ : calcd for $\mathrm{C}_{13} \mathrm{H}_{14} \mathrm{~N}_{3}[\mathrm{M}+\mathrm{H}]^{+}$ 212.1182, found 212.1182.

目标化合物 $\mathbf{2 b} \sim \mathbf{2 m}$ 的合成通法: 将 $43 \mathrm{mg}(0.2$ $\mathrm{mmol}$ )中间体化合物 $\mathbf{1}$ 溶解于 $5 \mathrm{~mL}$ 无水乙醇中, 加入 $0.22 \mathrm{mmol}$ 肼衍生物, 加热回流 $2 \sim 3 \mathrm{~h}$, 冷却, 反应混合 物旋蒸浓缩, 粗产品用柱层析 $[V$ (乙酸乙酯) $: V($ 甲苯 $)=$ 1:5]分离、纯化, 得到纯的目标产物, 收率 $60 \% \sim 86 \%$.

2-(1-苯基- $1 H$-吡唑-基)-3,3-二甲基-3H-吲哚(2b): 红色晶体，收率 $85 \%$. $R_{\mathrm{f}}=0.63 ;{ }^{1} \mathrm{H}$ NMR $\left(\mathrm{CDCl}_{3}, 400\right.$ $\mathrm{MHz}) \delta: 1.72(\mathrm{~s}, 6 \mathrm{H}), 7.40 \sim 7.42(\mathrm{~m}, 2 \mathrm{H}), 7.46 \sim 7.49(\mathrm{~m}$, 2H), $7.52(\mathrm{t}, J=8.0 \mathrm{~Hz}, 2 \mathrm{H}), 7.88(\mathrm{dd}, J=4.0,8.0 \mathrm{~Hz}$, $3 \mathrm{H}), 8.45(\mathrm{~s}, 1 \mathrm{H}), 10.05(\mathrm{~s}, 1 \mathrm{H}) ;{ }^{13} \mathrm{C} \mathrm{NMR}(75 \mathrm{MHz}$, $\left.\mathrm{CDCl}_{3}\right) \delta: 26.8(2), 54.3,119.5,121.2(2), 123.0,123.1$, $128.9,129.0,129.8,130.4,131.2(2), 133.8,140.0,142.9$, 144.3, 179.54; ESI-MS $m / z: 288.2[\mathrm{M}+\mathrm{H}]^{+}$. 数据同文献 [10].

2-[(3-甲基苯基)- $1 H$-吡唑-基]-3,3-二甲基-3H-吲哚 (2c): 黄色糖浆状物, 收率 $86 \% . R_{\mathrm{f}}=0.68 ;{ }^{1} \mathrm{H} \mathrm{NMR}$ $\left(\mathrm{CDCl}_{3}, 400 \mathrm{MHz}\right) \delta: 1.47(\mathrm{~s}, 6 \mathrm{H}), 2.35$ (s, 3H), 7.07 (d, $J=8.0 \mathrm{~Hz}, 1 \mathrm{H}), 7.16(\mathrm{t}, J=8.0 \mathrm{~Hz}, 1 \mathrm{H}), 7.28(\mathrm{dd}, J=4.0$, $8.0 \mathrm{~Hz}, 3 \mathrm{H}), 7.49$ (d, $J=4.0 \mathrm{~Hz}, 1 \mathrm{H}), 7.58$ (t, $J=8.0 \mathrm{~Hz}$, $2 \mathrm{H}), 8.28(\mathrm{~s}, 1 \mathrm{H}), 8.59(\mathrm{~s}, 1 \mathrm{H}) ;{ }^{13} \mathrm{C} \mathrm{NMR}(75 \mathrm{MHz}$, $\left.\mathrm{CDCl}_{3}\right) \delta: 22.8,26.1(2), 54.6,117.9,119.4,121.4,121.6$, $122.4,126.8,128.7,129.3,129.5,130.8,140.8,141.1$, 142.0, 147.6, 154.9, 179.7; ESI-MS m/z: $302.2[\mathrm{M}+\mathrm{H}]^{+}$. 数据同文献[10].

2-[(4-甲基苯基)- $1 H$-吡唑-基]-3,3-二甲基-3H-吲哚 (2d): 浅黄色固体，收率 $86 \% . R_{\mathrm{f}}=0.68 ;{ }^{1} \mathrm{H}$ NMR $\left(\mathrm{CDCl}_{3}, 400 \mathrm{MHz}\right) \delta: 1.55(\mathrm{~s}, 6 \mathrm{H}), 2.40(\mathrm{~s}, 3 \mathrm{H}), 7.23(\mathrm{~d}$, $J=8.0 \mathrm{~Hz}, 1 \mathrm{H}), 7.28(\mathrm{~d}, J=8.0 \mathrm{~Hz}, 2 \mathrm{H}), 7.35$ (dd, $J=4.0$, $8.0 \mathrm{~Hz}, 2 \mathrm{H}), 7.65(\mathrm{~d}, J=8.0 \mathrm{~Hz}, 3 \mathrm{H}), 8.30$ (s, 1H), 8.65 (s, $1 \mathrm{H}) ;{ }^{13} \mathrm{C} \mathrm{NMR}\left(75 \mathrm{MHz}, \mathrm{CDCl}_{3}\right) \delta: 22.4,26.2(2)$, 54.6, $119.1,120.9(2), 121.4,122.4,126.8,128.7,129.4$, 131.6(2), 138.4, 138.7, 141.9, 147.5, 154.6, 179.7; ESI-MS $m / z: 302.2[\mathrm{M}+\mathrm{H}]^{+}$. HRMS calcd for $\mathrm{C}_{20} \mathrm{H}_{20} \mathrm{~N}_{3}[\mathrm{M}+\mathrm{H}]^{+}$ 302.1652 , found 302.1650 .

2-[(3-甲氧基苯基)- $1 H$-吡唑-基]-3,3-二甲基- $3 H$-吲 哚 $(2 \mathrm{e})$ : 红色糖浆状物, 收率 $86 \% . R_{\mathrm{f}}=0.66 ;{ }^{1} \mathrm{H} \mathrm{NMR}$ $\left(\mathrm{CDCl}_{3}, 400 \mathrm{MHz}\right) \delta: 1.51(\mathrm{~s}, 6 \mathrm{H}), 3.82(\mathrm{~s}, 3 \mathrm{H}), 6.84(\mathrm{~d}$, $J=4.0 \mathrm{~Hz}, 1 \mathrm{H}), 7.22$ (ddd, $J=4.0,8.0,12.0 \mathrm{~Hz}, 1 \mathrm{H})$, $7.30 \sim 7.32(\mathrm{~m}, 3 \mathrm{H}), 7.33 \sim 7.35(\mathrm{~m}, 2 \mathrm{H}), 7.62(\mathrm{~d}, J=8.0$ $\mathrm{Hz}, 1 \mathrm{H}), 8.29(\mathrm{~s}, 1 \mathrm{H}), 8.68(\mathrm{~s}, 1 \mathrm{H}) ;{ }^{13} \mathrm{C}$ NMR $(75 \mathrm{MHz}$, 
$\left.\mathrm{CDCl}_{3}\right) \delta:$ 26.2(2), 47.2, 57.0, 106.7, 112.8, 114.8, 119.0, 121.3, 122.5, 127.0, 129.1, 129.4, 131.8(2), 142.0, 147.3, 154.2, 162.1, 179.6; ESI-MS $m / z: 318.3[\mathrm{M}+\mathrm{H}]^{+}$. HRMS calcd for $\mathrm{C}_{20} \mathrm{H}_{20} \mathrm{~N}_{3} \mathrm{O}[\mathrm{M}+\mathrm{H}]^{+}$318.1601, found 318.1605.

2-[1-(4-甲氧基苯基)- $1 \mathrm{H}$-吡唑-基]-3,3-二甲基- $3 \mathrm{H}$ 吲哚 $(2 \mathrm{f})$ : 深红色晶体, 收率 $86 \% . R_{\mathrm{f}}=0.55 ;{ }^{1} \mathrm{H}$ NMR $\left(\mathrm{CDCl}_{3}, 400 \mathrm{MHz}\right) \delta: 1.60(\mathrm{~s}, 6 \mathrm{H}), 3.86(\mathrm{~s}, 3 \mathrm{H}), 7.02(\mathrm{~d}$, $J=8.0 \mathrm{~Hz}, 2 \mathrm{H}), 7.30$ (d, $J=8.0 \mathrm{~Hz}, 1 \mathrm{H}), 7.39$ (t, $J=8.0$ $\mathrm{Hz}, 2 \mathrm{H}), 7.71(\mathrm{~d}, J=8.0 \mathrm{~Hz}, 3 \mathrm{H}), 8.32(\mathrm{~s}, 1 \mathrm{H}), 8.91(\mathrm{~s}$, $1 \mathrm{H}) ;{ }^{13} \mathrm{C}$ NMR (75 MHz, $\mathrm{CDCl}_{3}$ ) $\delta: 24.8(2), 52.4,55.6$, $114.7(2), 116.0,119.2,121.0,121.2(2), 125.9,128.2$, 128.7, 132.8, 140.6, 145.1, 149.7, 158.8, 178.4; ESI-MS $m / z: 318.3[\mathrm{M}+\mathrm{H}]^{+}$. 数据同文献[10].

2-[1-(2-硝基苯基)- $1 H$-吡唑-基]-3,3-二甲基-3H-吲 哚(2g): 浅红色晶体, 收率 $67 \% . R_{\mathrm{f}}=0.42 ;{ }^{1} \mathrm{H} \mathrm{NMR}$ $\left(\mathrm{CDCl}_{3}, 400 \mathrm{MHz}\right) \delta: 1.54(\mathrm{~s}, 6 \mathrm{H}), 7.26(\mathrm{dd}, J=4.0,8.0$ $\mathrm{Hz}, 1 \mathrm{H}), 7.36$ (t, $J=8.0 \mathrm{~Hz}, 2 \mathrm{H}), 7.59(\mathrm{t}, J=8.0 \mathrm{~Hz}, 1 \mathrm{H})$, 7.66 (dd, $J=4.0,8.0 \mathrm{~Hz}, 2 \mathrm{H}), 7.74(\mathrm{t}, J=8.0 \mathrm{~Hz}, 1 \mathrm{H}), 7.95$ (d, $J=4.0 \mathrm{~Hz}, 1 \mathrm{H}), 8.34(\mathrm{~s}, 1 \mathrm{H}), 8.42(\mathrm{~s}, 1 \mathrm{H}) ;{ }^{13} \mathrm{C} \mathrm{NMR}$ $\left(75 \mathrm{MHz}, \mathrm{CDCl}_{3}\right) \delta: 26.0(2), 54.7,120.0,121.7,122.4$, $126.8,127.0,127.9,129.4,130.6,131.6,134.3,134.7$, 143.1, 145.8, 147.6, 155.0, 179.1; ESI-MS $\mathrm{m} / \mathrm{z}: 333.3$ $[\mathrm{M}+\mathrm{H}]^{+}$. HRMS calcd for $\mathrm{C}_{19} \mathrm{H}_{17} \mathrm{~N}_{4} \mathrm{O}_{2}[\mathrm{M}+\mathrm{H}]^{+}$ 333.1346, found 333.1347.

2-[1-(3-硝基苯基)- $1 \mathrm{H}$-吡唑-基]-3,3-二甲基-3H-吲 哚(2h): 紫红色固体, 收率 $62 \% . R_{\mathrm{f}}=0.58 ;{ }^{1} \mathrm{H}$ NMR $\left(\mathrm{CDCl}_{3}, 400 \mathrm{MHz}\right) \delta: 1.58(\mathrm{~s}, 6 \mathrm{H}), 7.29(\mathrm{~d}, J=8.0 \mathrm{~Hz}$, $1 \mathrm{H}), 7.38(\mathrm{dd}, J=4.0,8.0 \mathrm{~Hz}, 2 \mathrm{H}), 7.69$ (dd, $J=8.0,12.0$ $\mathrm{Hz}, 2 \mathrm{H}), 8.20$ (t, $J=8.0 \mathrm{~Hz}, 2 \mathrm{H}), 8.38(\mathrm{~s}, 1 \mathrm{H}), 8.68(\mathrm{~s}, 1 \mathrm{H})$, $8.80(\mathrm{~s}, 1 \mathrm{H}) ;{ }^{13} \mathrm{C}$ NMR $\left(75 \mathrm{MHz}, \mathrm{CDCl}_{3}\right) \delta: 26.0(2), 54.7$, $115.6,121.7,122.5,123.1,126.1,127.3,128.4,128.7$, 129.5, 132.1, 141.4, 141.7, 147.0, 143.0, 150.3, 179.0; ESI-MS $m / z: 333.2[\mathrm{M}+\mathrm{H}]^{+}$. HRMS calcd for $\mathrm{C}_{19} \mathrm{H}_{17} \mathrm{~N}_{4} \mathrm{O}_{2}[\mathrm{M}+\mathrm{H}]^{+}$333.1346, found 333.1341.

2-[1-(2,4-二硝基苯基)- $1 H$-吡唑-基]-3,3-二甲基- $3 H$ 吲哚(2i): 黄色固体, 收率 $60 \% . R_{\mathrm{f}}=0.56 ;{ }^{1} \mathrm{H} \mathrm{NMR}$ $\left(\mathrm{CDCl}_{3}, 400 \mathrm{MHz}\right) \delta: 1.58(\mathrm{~s}, 6 \mathrm{H}), 7.32(\mathrm{t}, J=8.0 \mathrm{~Hz}, 1 \mathrm{H})$, 7.40 (t, $J=8.0 \mathrm{~Hz}, 2 \mathrm{H}), 7.70$ (d, $J=8.0 \mathrm{~Hz}, 1 \mathrm{H}), 7.97$ (s, $1 \mathrm{H}), 8.41(\mathrm{~s}, 1 \mathrm{H}), 8.59(\mathrm{~s}, 1 \mathrm{H}), 8.77(\mathrm{~s}, 1 \mathrm{H}), 8.79(\mathrm{~s}, 1 \mathrm{H})$; ${ }^{13} \mathrm{C}$ NMR (75 MHz, $\mathrm{CDCl}_{3}$ ) $\delta: 24.6(2), 53.1,117.1,120.4$, $121.1,121.3,126.2,126.4,127.8,128.2,128.3,130.6$, $140.4,143.1,145.6,146.2,149.3,178.1$. ESI-MS $m / z$ : $378.2[\mathrm{M}+\mathrm{H}]^{+}$. HRMS calcd for $\mathrm{C}_{19} \mathrm{H}_{16} \mathrm{~N}_{5} \mathrm{O}_{4}[\mathrm{M}+\mathrm{H}]^{+}$ 378.1197 , found 378.1197 .

2-[1-(2-三氟甲基苯基)-1 $H$-吡唑-基]-3,3-二甲基-
$3 H$-吲槑 $(\mathbf{2 j})$ : 浅黄色固体, 收率 $85 \% . R_{\mathrm{f}}=0.47 ;{ }^{1} \mathrm{H} \mathrm{NMR}$ $\left(\mathrm{CDCl}_{3}, 400 \mathrm{MHz}\right) \delta: 1.53(\mathrm{~s}, 6 \mathrm{H}), 7.26(\mathrm{t}, J=8.0 \mathrm{~Hz}, 1 \mathrm{H})$, $7.35(\mathrm{t}, J=8.0 \mathrm{~Hz}, 2 \mathrm{H}), 7.61(\mathrm{t}, J=8.0 \mathrm{~Hz}, 2 \mathrm{H}), 7.65(\mathrm{~d}$, $J=8.0 \mathrm{~Hz}, 1 \mathrm{H}), 7.71(\mathrm{t}, J=8.0 \mathrm{~Hz}, 1 \mathrm{H}), 7.84(\mathrm{~d}, J=8.0$ $\mathrm{Hz}, 1 \mathrm{H}), 8.34$ (s, 1H), 8.40 (s, 1H); ${ }^{13} \mathrm{C}$ NMR $(75 \mathrm{MHz}$, $\mathrm{CDCl}_{3}$ ) $\delta: 26.0(2), 54.7,118.9,121.6,122.4,125.7,126.8$, $128.7,128.8,129.3,130.4,130.8,133.4,134.4,139.4$, 142.4, 147.6, 155.0, 179.4; ESI-MS $m / z$ : $356.2[\mathrm{M}+\mathrm{H}]^{+}$. HRMS calcd for $\mathrm{C}_{20} \mathrm{H}_{17} \mathrm{~N}_{3} \mathrm{~F}_{3}[\mathrm{M}+\mathrm{H}]^{+} 356.1369$, found 356.1370 .

2-[1-(3-三氟甲基苯基)-1 $\mathrm{H}$-吡唑-基]-3,3-二甲基$3 H$-吲哚 $(\mathbf{2 k})$ : 红色糖浆状物, 收率 $86 \% . R_{\mathrm{f}}=0.71 ;{ }^{1} \mathrm{H}$ NMR $\left(\mathrm{CDCl}_{3}, 400 \mathrm{MHz}\right) \delta: 1.56(\mathrm{~s}, 6 \mathrm{H}), 7.26$ (t, $J=8.0$ $\mathrm{Hz}, 1 \mathrm{H}), 7.36(\mathrm{~m}, 2 \mathrm{H}), 7.61(\mathrm{~d}, J=4.0 \mathrm{~Hz}, 2 \mathrm{H}), 7.66(\mathrm{~d}$, $J=8.0 \mathrm{~Hz}, 1 \mathrm{H}), 7.98$ (d, $J=8.0 \mathrm{~Hz}, 1 \mathrm{H}), 8.11(\mathrm{~s}, 1 \mathrm{H}), 8.36$ (s, $1 \mathrm{H}), 8.78(\mathrm{~s}, 1 \mathrm{H}) ;{ }^{13} \mathrm{C}$ NMR $\left(75 \mathrm{MHz}, \mathrm{CDCl}_{3}\right) \delta$ : 26.0(2), 54.7, 117.8, 119.8, 121.5, 122.5, 123.6, 123.7, $125.2,125.3,127.2,128.8,129.4,131.7,141.2,142.6$, 147.4, 154.3, 179.3; ESI-MS m/z: $356.3[\mathrm{M}+\mathrm{H}]^{+}$. HRMS calcd for $\mathrm{C}_{20} \mathrm{H}_{17} \mathrm{~N}_{3} \mathrm{~F}_{3}[\mathrm{M}+\mathrm{H}]^{+}$356.1369, found 356.1369.

2-[1-(4-三氟甲基苯基)- $1 \mathrm{H}$-吡唑-基]-3,3-二甲基$3 H$-吲哚(2I): 浅黄色针状晶体, 收率 $60 \% . R_{\mathrm{f}}=0.76 ;{ }^{1} \mathrm{H}$ NMR $\left(\mathrm{CDCl}_{3}, 400 \mathrm{MHz}\right) \delta$ : 1.58 (s, 6H), 7.30 (d, $J=8.0$ $\mathrm{Hz}, 1 \mathrm{H}), 7.39$ (t, $J=8.0 \mathrm{~Hz}, 2 \mathrm{H}), 7.68(\mathrm{~d}, J=4.0 \mathrm{~Hz}, 1 \mathrm{H})$, 7.78 (d, $J=8.0 \mathrm{~Hz}, 2 \mathrm{H}), 7.94(\mathrm{~d}, J=8.0 \mathrm{~Hz}, 2 \mathrm{H}), 8.36$ (s, $1 \mathrm{H}), 8.91(\mathrm{~s}, 1 \mathrm{H}) ;{ }^{13} \mathrm{C} \mathrm{NMR}\left(75 \mathrm{MHz}, \mathrm{CDCl}_{3}\right) \delta: 25.1(2)$, 53.2, 116.0, 119.1, 119.6(2), 121.5, 126.9, 127.0, 127.1(2), 128.6, 129.1, 130.4, 141.1, 141.6, 144.1, 147.7, 177.7; ESI-MS $m / z$ : $356.3[\mathrm{M}+\mathrm{H}]^{+}$. HRMS calcd for $\mathrm{C}_{20} \mathrm{H}_{17} \mathrm{~N}_{3} \mathrm{~F}_{3}$ $[\mathrm{M}+\mathrm{H}]^{+} 356.1369$, found 356.1369 .

2-[1-(3,5-双三氟甲基苯基)- $1 H$-吡唑-基]-3,3-二甲 基-3H-吲哚(2m): 黄色针状晶体, 收率 $60 \% . R_{\mathrm{f}}=0.84$; ${ }^{1} \mathrm{H} \mathrm{NMR}\left(\mathrm{CDCl}_{3}, 400 \mathrm{MHz}\right) \delta: 1.59$ (s, 6H), 7.30 (t, $J=8.0$ $\mathrm{Hz}, 1 \mathrm{H}), 7.39$ (dd, $J=4.0,8.0 \mathrm{~Hz}, 2 \mathrm{H}), 7.69$ (d, $J=8.0 \mathrm{~Hz}$, $1 \mathrm{H}), 7.86(\mathrm{~s}, 1 \mathrm{H}), 8.29(\mathrm{~s}, 2 \mathrm{H}), 8.38(\mathrm{~s}, 1 \mathrm{H}), 8.88(\mathrm{~s}, 1 \mathrm{H})$; ${ }^{13} \mathrm{C}$ NMR (75 MHz, $\mathrm{CDCl}_{3}$ ) $\delta: 26.0(2), 54.7,120.6,121.5$, $122.0,122.5(2), 125.5,125.6,127.4,129.6(2), 134.2$, 134.5, 134.9, 141.7, 143.1(2), 144.1, 178.8; ESI-MS $m / z$ : $424.2[\mathrm{M}+\mathrm{H}]^{+}$. HRMS calcd for $\mathrm{C}_{21} \mathrm{H}_{16} \mathrm{~N}_{3} \mathrm{~F}_{6}[\mathrm{M}+\mathrm{H}]^{+}$ 424.1243, found 424.1245 .

\subsubsection{MTT 法检测肿瘤细胞存活率}

利用 MTT 法篮选该类化合物的抗肿瘤活性. 实 验过程: 细胞培养至对数生长期, 用 $0.25 \%$ 胰蛋白酶消 化, 之后, 细胞悬液接种到 96 孔板中, 细胞浓度为 $5 \times$ $10^{3}$ 个账. 平板置于 $37{ }^{\circ} \mathrm{C}, 5 \% \mathrm{CO}_{2}$ 及饱和湿度培养箱中 
培养 24 h. 之后将不同浓度的样品溶液加入到 96 孔板 中, 培养 $72 \mathrm{~h}$. 之后加入 $30 \mu \mathrm{L}$ MTT $[5 \mathrm{mg} / \mathrm{mL}$ in PBS(磷酸钾缓冲溶液)], 继续培养 $4 \mathrm{~h}$. 吸掉上层培养 液, 加入 $100 \mu \mathrm{L} \mathrm{DMSO}$, 微震荡 $10 \mathrm{~min}$ 后, 用酶标仪 (Thermo MK3, USA)检测 $570 \mathrm{~nm}$ 处的吸光度值. 化合 物初篎浓度为 50 和 $5 \mu \mathrm{mol} / \mathrm{L}$. 细胞对照组: 只加细胞, 不加药物; 阳性对照组: 长春碱; 空白对照组: 不加细 胞, 不加培养液. 细胞存活率 $(\%)=($ 加药孔一空白 孔)/(正常细胞孔一空白孔 $) \times 100 \%$.

\section{References}

[1] Noble, R. L.; Beer, C. T.; Cutts, J. H. Ann. N. Y. Acad. Sci. 1958, 76,882 .

[2] Svoboda, G. H.; Neuss, N.; Gorman, M. J. J. Am. Pharm. Assoc. 1959, 48,659

[3] Dancey, J.; Steward, W. P. Anti-cancer Drugs 1995, 6, 625.

[4] (a) Langlois, N.; Gueritte, F.; Langlois, Y.; Potier, P. J. Am. Chem. Soc. 1976, 98, 7017.

(b) Cros, S.; Wright, M.; Morimoto, M.; Lataste, H.; Couzinier, J. P.; Krikorian, A. Semin. Oncol. 1989, 16, 15.

[5] Sasaki, Y.; Kato, D.; Boger, D. L. J. Am. Chem. Soc. 2010, 132, 13533.
[6] Schleicher, K. D.; Sasaki, Y.; Tam, A. Kato, D.; Duncan, K. K.; Boger, D. L. J. Med. Chem. 2013, 56, 483.

[7] Keglevich, P.; Hazai, L.; Kalaus, G.; Szántay, C. Molecules 2012 , $17,5893$.

[8] Ngo, Q. A.; Roussi, F.; Thoret, S.; Guéritte, F. Chem. Biol. Drug Des. 2010, 75, 284.

[9] Zheng, J.; Deng, L. J.; Chen, M. F.; Xiao, X. Z.; Xiao, S. W.; Guo, C. P.; Xiao, G. K.; Bai, L. L.; Ye, W. C.; Zhang, D. M.; Chen, H. R. Eur. J. Med. Chem. 2013, 65, 158.

[10] Baradarani, M. M.; Afghan, A.; Zebarjadi, F.; Hasanzadeh, K.; Joule, J. A. J. Heterocycl. Chem. 2006, 43, 1591.

[11] Rashidi, A.; Afghan, A.; Baradarani, M. M.; Joule, J. A. J. Heterocycl. Chem. 2009, 46, 428.

[12] Ye, J. H.; Ye, W. F.; Xiao, C. T.; Chen, Y.; Wang, G. P.; Zhang, W. Chin. J. Org. Chem. 2012, 32, 1503 (in Chinese). (叶家海, 叶文芳, 肖承涛, 陈 雨, 王光普, 张文超, 有机化学, 2012, 32, 1503.)

[13] Kumar, V.; Kaur, K.; Karelia, D. N.; Beniwal, V.; Gupta, G. K.; Sharma, A. K.; Gupta, A. K. Eur. J. Med. Chem. 2014, 81, 267.

[14] Zhang, D. M.; Liu, J. S.; Tang, M. K.; Liu, A.; Cao, H. H.; Jiang, L.; Chan, J. Y.; Tian, H. Y.; Fung, K. P.; Ye, W. C. Eur. J. Pharmacol. 2012, 692, 19.

[15] Gigant, B.; Wang, C. G.; Ravelli, R. B. G.; Roussi, F.; Steinmetz, M. O.; Curmi, P. A.; Sobel, A.; Knossow, M. Nature 2005, 435, 519.

(Qin, X.) 